\title{
ELEMENTARY STEPS IN ENZYME CATALYSIS AND REGULATION
}

\author{
GORDON G. HAMMES
}

Department of Chemistry, Cornell University, Ithaca, New York 14850,
USA

ABSTRACT

A general approach to elucidating the molecular basis of enzyme catalysis and regulation is the time resolution of the complex reaction mechanisms involved into their elementary steps. Both enzyme catalysis and enzyme regulation are initiated by the binding of small molecules to the protein. This association reaction is generally quite rapid and is often followed by a conformational change of the macromolecule. These conformational changes are discussed in terms of the dynamics of the underlying elementary processes, hydrogen bonding, solvation and hydrophobic interactions, all of which have been studied in model systems. Enzyme catalysis often involves acid-base catalysis. Consideration of the rates of both concerted and sequential proton transfer mechanisms in terms of model systems suggests that the observed turnover numbers of enzymes require that proton transfer steps in the catalytic process occur at close to their maximum possible rates. A general mechanism for enzyme catalysis is proposed and is illustrated by the mechanism of action of ribonuclease A. A distinguishing feature of most regulatory enzymes is their multi-subunit nature. The regulation of enzyme activity usually is achieved by alteration of subunit interactions through conformational changes triggered by ligand binding. These conformational transitions are important processes in the cooperative binding of substrates and effectors to regulatory enzymes. As an example, the mechanism of the regulation of aspartate transcarbamylase from Escherichia coli is considered: in this case coupled conformational changes appear to be utilized as interlocking on-off switches for the enzymic activity.

\section{INTRODUCTION}

Enzymes have two important physiological functions : efficient catalysis of metabolic reactions and regulation of metabolic processes. In this presentation the elementary steps and molecular bases of these two functions are considered.

The most remarkable features of enzyme catalysis are the very high efficiency and great specificity relative to model systems. For example, the enzyme fumarase, which catalyses the hydration of fumarate to L-malate, has a specific catalytic rate constant of about $2 \times 10^{3} \mathrm{~s}^{-1}$ at $25^{\circ}$ when saturated with substrate ${ }^{1}$, while the same reaction in $1 \mathrm{M}$ hydrogen and hydroxide ion has specific rate constants of approximately $2 \times 10^{-8} \mathrm{~s}^{-1}$ and $2 \times 10^{-9}$ $\mathrm{s}^{-1}$, respectively ${ }^{2,3}$. Moreover, no other substrates for this enzyme have been found other than fumarate and L-malate, except for cases where fluorine 
has been substituted for some of the substrate hydrogens. Although the mechanism of enzyme action has been actively studied for many years, many of the molecular details remain to be delineated.

The regulation of enzymes is a physiological process which does not yet have a true parallel in model systems. The turning on and off of enzymatic activity by specific molecules is crucial for the control of metabolic fluxes. For example, aspartate transcarbamylase, which catalyses the carbamylation of aspartic acid by carbamyl phosphate, is inhibited by the ultimate endproduct of the biosynthetic pathway, cytidine-5'-triphosphate (CTP), which effectively shuts down pyrimidine biosynthesis; this same enzyme is activated (turned on) by the purine, adenosine- $5^{\prime}$-triphosphate (ATP) ${ }^{4}$. A second example of an enzyme with important regulatory properties is phosphofructokinase, which plays a central role in glycolysis and catalyses the transfer of a phosphoryl group from adenosine-5'-triphosphate to fructose-6-phosphate to give fructose-1,6-diphosphate and the nucleotide diphosphate. It is activated by a variety of substances, including phosphate, fructose-1,6diphosphate, adenosine-5'-monophosphate and adenosine-5'-diphosphate, and is inhibited by several metabolites, including citrate and magnesium adenosine-5'-triphosphate ${ }^{5,6}$. The regulation of enzymes is also controlled at the genetic level where the synthesis of enzymes can be turned on and off, but this important aspect of regulation will not be discussed.

The approaches to understanding enzyme catalysis stressed here are thermodynamics and kinetics. Basically this means that the chemical processes are studied as a function of concentrations and time. It is important that the accessible time range be as broad as possible in order that all of the individual elementary steps can be isolated and studied. At the present time methods are available, such as magnetic resonance, ultrasonic attenuation measurements, the temperature jump method and stopped flow techniques, which permit reaction time constants as short as $10^{-10}-10^{-11} \mathrm{~s}$ to be measured (cf. reference 7). A summary of currently available experimental techniques for kinetic studies of enzyme reactions and their approximate time resolution is given in Table 1 . Since molecular vibrations occur in $10^{-12}$ $10^{-13} \mathrm{~s}$, virtually the entire time range of chemical events is accessible. The delineation of the elementary steps in enzymatic processes permits the development of formal kinetic models, which ultimately must be interpreted in structural terms.

In the discussion to be presented here the common elementary steps involved in catalysis and regulation are considered. both in terms of reactions occurring in the actual enzymatic process and in terms of model reactions. Relaxation methods are particularly useful in analysing complex

Table 1. Fast reaction techniques for the study of enzymes

\begin{tabular}{|c|c|c|c|}
\hline Technique & $\begin{array}{l}\text { Applicable time } \\
\text { range (s) }\end{array}$ & Technique & $\begin{array}{l}\text { Applicable time } \\
\text { range (s) }\end{array}$ \\
\hline Nuclear magnetic resonance & $10^{-6}->1$ & Temperature jump & $10^{-8}->1$ \\
\hline Electron magnetic resonance & $10^{-5}-10^{-10}$ & Pressure jump & $10^{-6}->1$ \\
\hline Rapid mixing & $10^{-4}->1$ & Acoustic methods & $10^{-3}-10^{-11}$ \\
\hline
\end{tabular}


mechanisms, since the number of relaxation processes or time constants (relaxation times) observed is a direct measure of the minimum number of steps in the mechanism. Two particular enzyme mechanisms will be discussed in detail: the breakdown of ribonucleic acids by ribonuclease and the regulation of enzyme catalysis by aspartate transcarbamylase.

\section{INITIATION OF CATALYSIS AND REGULATION}

The initiation of catalysis and of regulation is similar, namely the binding of a substrate or effector (i.e. regulator) molecule to the enzyme. The rate of binding of many small molecules to enzymes, including substrates, inhibitors and regulatory ligands, has been studied with fast reaction techniques (cf. references 8 and 9). Some representative data are presented in Table 2. The

Table 2. Representative rate constants for the reaction $\mathrm{E}+\mathrm{L} \underset{k_{\mathrm{r}}}{\stackrel{k_{\mathrm{r}}}{-}} \mathrm{EL}$

\begin{tabular}{|c|c|c|c|c|}
\hline Enzyme (E) & Ligand (L) & $\begin{array}{c}10^{-7} k_{\mathrm{f}} \\
\left(\mathrm{M}^{-1} \mathrm{~s}^{-1}\right)\end{array}$ & $k_{\mathrm{r}}\left(\mathrm{s}^{-1}\right)$ & Reference \\
\hline Chymotrypsin & $\begin{array}{l}\text { Furylacryloyl-L- } \\
\text { tryptophan amide }\end{array}$ & 0.62 & $2.7 \times 10^{3}$ & 10 \\
\hline \multirow[t]{5}{*}{ Ribonuclease } & Cytidine- $3^{\prime}$-phosphate & 4.6 & $4.2 \times 10^{3}$ & 11 \\
\hline & Uridine- $3^{\prime}$-phosphate & 7.8 & $1.1 \times 10^{4}$ & 11 \\
\hline & $\begin{array}{l}\text { Cytidine }-2^{\prime}, 3^{\prime} \text { cyclic } \\
\text { phosphate }\end{array}$ & $2-4$ & $1-2 \times 10^{4}$ & 12 \\
\hline & $\begin{array}{l}\text { Uridine- } 2^{\prime}, 3^{\prime} \text { cyclic } \\
\text { phosphate }\end{array}$ & 1 & $2 \times 10^{4}$ & 13 \\
\hline & Cytidylyl-3',5'-cytidine & 1.4 & $7 \times 10^{3}$ & 14 \\
\hline \multirow[t]{4}{*}{ Creatine kinase } & ADP & 2.2 & $1.8 \times 10^{4}$ & 15 \\
\hline & MgADP & 0.53 & $5.1 \times 10^{3}$ & 15 \\
\hline & $\mathrm{CaADP}$ & 0.17 & $1.2 \times 10^{3}$ & 15 \\
\hline & MnADP & 0.74 & $4.1 \times 10^{3}$ & 15 \\
\hline Lactate dehydrogenase & NADH & 5.46 & 39 & 16 \\
\hline Glyceraldehyde 3- & $\mathrm{NAD}^{a}$ & 1.1 & $1.1 \times 10^{3}$ & 17 \\
\hline $\begin{array}{l}\text { phosphate dehydro- } \\
\text { genase (yeast) }\end{array}$ & & 0.032 & $8 \times 10^{2}$ & \\
\hline
\end{tabular}

${ }^{a}$ Two types of binding sites are present.

second-order rate constants measured are typically in the range of $10^{7}$ $10^{8} \mathrm{M}^{-1} \mathrm{~s}^{-1}$. Thus, although the interaction of an enzyme with a substrate or effector is often very specific, with rigid stereochemical requirements, nevertheless the initial complex formation is very rapid and is close to being controlled by how rapidly the reactants diffuse together. The dissociation rate constant of the initial complex formed varies quite widely, and usually can be interpreted as a direct measure of the strength of the small moleculeenzyme interaction (i.e. it parallels the thermodynamic binding constants, since the association rate constants do not vary greatly). Another general feature of the interaction between enzyme and substrate or effector is that an isomerization of the complex often follows the binding - that is, the complex (and sometimes the unliganded enzyme) can exist in two or more different conformations. The rates of the conformational changes vary considerably, 
and some typical rates are presented in Table 3, together with the functional nature of the conformational changes. A wide range of time constants has been observed, from microseconds to minutes and even hours. Strong evidence exists that conformational transitions play a very important role in both catalysis and regulation. In the case of catalysis the rates of the conformational transitions must be more rapid than the rate of the over-all reaction, whereas quite slow transitions can be of importance in regulation. Although the function of the conformational changes differs in catalysis and regulation, the elementary steps involved are similar and will now be briefly discussed.

Table 3. Representative rates of protein conformational changes associated with ligand binding

\begin{tabular}{lcc} 
Enzyme & $\begin{array}{c}\text { Approximate } \\
\text { rate }\end{array}$ & Reference \\
\hline $\begin{array}{l}\text { Catalytic } \\
\text { Chymoirypsin }\end{array}$ & $10^{2} \mathrm{~s}^{-1}$ & 10 \\
Ribonuclease & $10^{3}-10^{4} \mathrm{~s}^{-1}$ & $11-14$ \\
Creatine kinase & $10^{4} \mathrm{~s}^{-1}$ & 15 \\
Glyceraldehyde-3-phosphate dehydrogenase (yeast) & $10^{4} \mathrm{~s}^{-1}$ & 17 \\
Aspartate transcarbamylase & $10^{3}-10^{4} \mathrm{~s}^{-1}$ & 18 \\
Regulatory & & \\
\hline Aspartate transcarbamylase & $10^{3}-10^{4} \mathrm{~s}^{-1}$ & 19,20 \\
& $10^{2}-10^{3} \mathrm{~s}^{-1}$ & 21 \\
Glyceraldehyde-3-phosphate dehydrogenase (yeast) & $10-10^{2} \mathrm{~s}^{-1}$ & 22 \\
Homoserine dehydrogenase $(E$. coli) & $1-10 \mathrm{~s}^{-1}$ & 17 \\
Threonine deaminase $(B$. subtilis) & $\min ^{-1}$ & 23 \\
& $\min ^{-1}$ & 24 \\
\hline
\end{tabular}

\section{ELEMENTARY STEPS IN CONFORMATIONAL CHANGES}

The basic processes in protein conformational changes involve noncovalent changes, primarily hydrogen bonding, solvation and hydrophobic interactions. These interactions are virtually inseparable in water, but information about each can be obtained from model systems. The dynamics of hydrogen bonding are difficult to study in water because water is such a good hydrogen bonding donor or acceptor. However, a number of kinetic studies of the formation of hydrogen-bond-stabilized dimers has been made in non-aqueous solvents. For example, the dimerization of 2-pyridone and benzoic acid, according to equations (1) and (2), has been extensively studied $^{25-29}$. These reactions are extremely rapid, with time constants in the range $10^{-7}-10^{-9} \mathrm{~s}$, and have been studied mainly through measurements of ultrasonic attenuation. Some typical rate constants for these reactions in a variety of solvents are summarized in Table 4 . The association rate constants

2

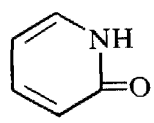

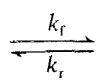

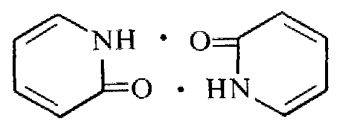


2<smiles>O=C(O)c1ccccc1</smiles>

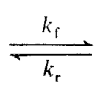

Table 4. Representative rate constants for hydrogen bond dimerization $2 \mathrm{~A} \underset{k_{\mathrm{r}}}{\stackrel{k_{\mathrm{f}}}{\rightleftharpoons}} \mathrm{A}$

\begin{tabular}{|c|c|c|c|c|}
\hline Reactant & Solvent & $\begin{array}{c}10^{-9} k_{\mathrm{f}} \\
\left(\mathrm{M}^{-1} \mathrm{~s}^{-1}\right)\end{array}$ & $\begin{array}{l}10^{-7} k_{\mathrm{r}} \\
\left(\mathrm{s}^{-1}\right)\end{array}$ & Reference \\
\hline \multirow[t]{3}{*}{ Benzoic acid } & $\mathrm{CCl}_{4}$ & 5.0 & 0.073 & 25 \\
\hline & $\mathrm{CHCl}_{3}$ & 4.7 & 0.75 & 26 \\
\hline & Hexane & 8.1 & 0.022 & 26 \\
\hline \multirow[t]{5}{*}{ 2-Pyridone } & $\mathrm{CHCl}_{3}$ & 3.3 & 2.2 & 27 \\
\hline & Dioxane & 2.1 & 13 & 28 \\
\hline & $1 \% \mathrm{H}_{2} \mathrm{O}$-dioxane & 1.7 & 17 & 28 \\
\hline & $\begin{array}{c}\mathrm{CCl}_{4} \text {-dimethyl } \\
\text { sulphoxide }(1.1 \mathrm{~m})\end{array}$ & 0.26 & 14.8 & 29 \\
\hline & $\begin{array}{c}\mathrm{CCl}_{4} \text {-dimethyl } \\
\text { sulphoxide }(5.5 \mathrm{~m})\end{array}$ & 0.069 & 27 & 29 \\
\hline
\end{tabular}

in all cases in Table 4, except for the last two entries, are approximately $10^{9}$ $\mathbf{M}^{-1} \mathbf{s}^{-1}$, which is essentially the value expected for a diffusion-controlled reaction. The corresponding dissociation rate constants, on the other hand, vary considerably and roughly parallel the thermodynamic stability of the hydrogen bonds. The mechanism of these reactions can be schematically written as follows :

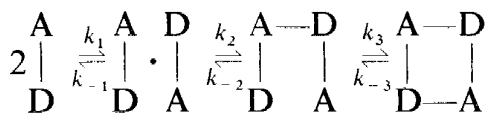

The first step in this mechanism represents the diffusion together of reactants; the second step represents the formation of the first hydrogen bond; and the third step represents the formation of the second hydrogen bond. If all of the intermediates are assumed to be present in a steady state, which is suggested by the fact that only a single time constant is found experimentally, the observed forward and reverse rate constants can be written as

$$
\begin{gathered}
k_{\mathrm{f}}=k_{1} /\left\{1+\left(k_{-1} / k_{2}\right)\left(1+k_{-2} / k_{3}\right)\right\} \\
k_{\mathrm{r}}=k_{-3} /\left\{1+\left(k_{3} / k_{-2}\right)\left(1+k_{2} / k_{-1}\right)\right\}
\end{gathered}
$$

In order for $k_{\mathrm{f}}$ to be equal to $k_{1}$, as indicated by the experimental data, $k_{2}$ must be greater than $k_{-1}$. In other words, desolvation of the solutes and formation of the first hydrogen bond must be faster than diffusion apart of the reactants. The value of $k_{-1}$ is about $10^{10} \mathrm{~s}^{-1}$, so that $k_{2}$ must be $10^{11}-10^{12}$ $\mathrm{s}^{-1}$, which is only $10-100$ molecular vibrations. The observed dissociation rate constant under these conditions is $k_{\mathrm{r}}=k_{-1}\left(k_{-2} / k_{2}\right)\left(k_{-3} / k_{3}\right)$, and since $k_{-1}$ is essentially the same for all cases, the reverse rate constant is a direct measure of the thermodynamic stability of solute-solute hydrogen bonds relative to solute-solvent hydrogen bonds. In solvents containing appre- 
ciable amounts of species forming strong hydrogen bonds, such as the last two entries in Table 4, where high concentrations of dimethylsulphoxide are present, the association rate is no longer diffusion-controlled. Instead a detailed kinetic analysis indicates that desolvation of the solute, with a specific rate constant of about $10^{8} \mathrm{~s}^{-1}$, is rate-determining ${ }^{29}$.

More direct measurements of desolvation rates have been made using both ultrasonic and n.m.r. techniques. Some typical rate constants are presented in Table 5. The dissociation of $\mathrm{H}_{2} \mathrm{O}$ from $\mathrm{NH}_{3}$ is diffusion-controlled; how-

Table 5. Representative desolvation rate constants

\begin{tabular}{lcc}
\hline Molecular species & $k\left(\mathrm{~s}^{-1}\right)$ & Reference \\
\hline $\mathrm{NH}_{3} \cdot \mathrm{H}_{2} \mathrm{O}$ & $2.2 \times 10^{11}$ & 30 \\
$\left(\mathrm{PhCH}_{3}\right)_{2} \mathrm{NCH}_{3} \cdot \mathrm{H}_{2} \mathrm{O}$ & $2.7 \times 10^{9}$ & 30 \\
Dioxane $\left(\mathrm{H}_{2} \mathrm{O}\right)_{2}$ & $2.8 \times 10^{8}$ & 31 \\
Dioxane $_{2}\left(\mathrm{H}_{2} \mathrm{O}\right)_{2}$ & $1.0 \times 10^{8}$ & 31 \\
Glycine, di, tri, glycine & $4 \times 10^{8}$ & 32 \\
\hline
\end{tabular}

${ }^{a}$ Only the sum of the solvation and desolvation rate constants, i.e. the reciprocal relaxation time, could be determined.

ever, as hydrophobic groups are placed around the hydrogen bond acceptor, the rate of dissociation of water decreases considerably. This is probably due to the fact that a sheath of strongly interacting water molecules forms around the hydrophobic groups, which dissociate more slowly. The conclusions to be derived from these studies which are relevant to proteins are that in a non-aqueous environment, such as might, for example, exist within a protein, the elementary step of hydrogen bond formation has a specific rate constant of $10^{11}-10^{12} \mathrm{~s}^{-1}$. The specific rate constant for desolvation of individual protein groups, which probably is often rate-limiting in hydrogen bond formation in water, is about $10^{8} \mathrm{~s}^{-1}$. Both of these rate constants suggest that the rate of conformational transitions in a protein should be considerably faster than those observed, and some additional studies with model systems suggest why this may be the case.

Ultrasonic measurements in aqueous polyethylene glycol solutions indicate that a relaxation process occurs with a reciprocal relaxation time of about $10^{8} \mathrm{~s}^{-133}$. This relaxation process is due to solvation equilibria coupled to the polymer chain motions or, in other words. the dynamics of hydrophobic and hydrogen bonding interactions involving solvent and polymer are being observed. The molecular weight dependence of the relaxation time is quite striking: the relaxation time increases with increasing molecular weight until a molecular weight of about 4000 is reached and then remains essentially constant (at $6 \times 10^{-9} \mathrm{~s}$ ) as the molecular weight is further increased ${ }^{34}$. This indicates that a molecular weight of about 4000 represents a maximum size unit for the relaxation process. Furthermore, with polymers of molecular weight greater than about 4000 , but not with very small polymers, the relaxation time decreases over a very narrow range of urea or guanidine concentration, which suggests that a cooperative change in solvent-polymer structure is occurring ${ }^{35,36}$. These results suggest that a minimum molecular size (in this case about 4000 molecular weight) is required for cooperativity, and the ultrasonic relaxation time for the solvent-polymer system increases 
as the degree of cooperativity increases. The obvious implication of these findings for proteins is that a possible rationale for the large size of proteins is to permit the occurrence of cooperative conformational transitions, and furthermore the slowness of the conformational transitions in proteins, relative to the rates of the elementary steps involved, is due to the fact they are highly cooperative. Both of these points are even more strongly illustrated by a second model system, polyglutamic acid. This polymer can exist in either a helical or random coil configuration, and a cooperative transition between these two states in aqueous solution can be triggered by small changes in $\mathrm{pH}$. This cooperative transition is observed only with polymers containing more than six residues ${ }^{37}$. Furthermore, the relaxation time for this process at the midpoint of the transition is only about $1 \mu \mathrm{s}^{38}$. The rate constant for the elementary step in helix formation has been estimated from theory and experiment to be about $8 \times 10^{7} \mathrm{~s}^{-138}$. Although hydrogen bonding has usually been assumed to be the dominant factor in helix formation, the magnitude of this rate constant suggests that desolvation is more likely to be the ratedetermining step.

These model studies indicate that the practical limitation on the rate constants for conformational changes in terms of the elementary steps involved in hydrogen bonding and solvation processes is about $10^{8} \mathrm{~s}^{-1}$. The fact that much slower conformational changes are observed (Table 3) suggests that highly cooperative transitions are occurring. Furthermore, highly cooperative phenomena require a large number of cooperative elements or, in molecular terms, a macromolecule.

\section{CATALYSIS}

A molecular explanation of the tremendous catalytic efficiency of enzymes remains an elusive goal for chemists. The actual bond-breaking and bondforming steps often involve acid-base catalysis, so that the elementary steps are proton transfer reactions. Proton transfer reactions have been extensively studied, so that it is possible to predict the rates of protolytic reactions with a great deal of certitude (cf. reference 39). For 'normal' acids and bases protonation and deprotonation with hydroxyl ion are diffusion-controlled processes with typical rate constants of $10^{10} \mathrm{M}^{-1} \mathrm{~s}^{-1}$. These processes can be written as

$$
\begin{aligned}
\mathrm{B}+\mathrm{H}^{+} & \rightleftharpoons \mathrm{BH}^{+} \\
\mathrm{BH}^{+}+\mathrm{OH}^{-} & \rightleftharpoons \mathrm{B}+\mathrm{H}_{2} \mathrm{O}
\end{aligned}
$$

By analogy with the earlier discussion of hydrogen bonding, the fact that these rates are diffusion-controlled implies that the actual proton transfer is fast compared with diffusion apart of the reactants - that is, the specific rate constant for intramolecular proton transfer in water is about $10^{12} \mathrm{~s}^{-1}$. This rate is very fast because of the rapid proton conduction which can occur through structured water. Marked deviations from diffusion control occur if the water structure is perturbed-for example, by internal hydrogen bonding or by an unusually high charge density.

In catalytic reactions the acid or base involved in catalysis must end up in 
the same state of protonation as it starts in. Thus, for solvent-mediated reactions, the cycle of equations (6) and (7) must occur. The rate constants for the reverse reactions can be readily calculated from the ionization constant of the acid and the fact that both of the forward rate constants are approximately $10^{10} \mathrm{M}^{-1} \mathrm{~s}^{-1}$. The rate constant for the reverse of equation (6) is $10^{10}$ $K_{\mathrm{a}} \mathrm{s}^{-1}$, while that for equation (7) is $10^{10} K_{\mathrm{w}} / K_{\mathrm{a}}$, where $K_{\mathrm{a}}$ is the acid ionization constant and $K_{\mathrm{w}}$ is the ionization constant of water. The maximum catalytic rate then occurs when both of the rate constants for the reverse reactions are maximized. This occurs with a $\mathrm{p} K_{\mathrm{a}}$ of about 7 , which of course is typical of an imidazole residue. Imidazole has been implicated as being essential for catalysis in many enzymatic reactions. These results indicate that the maximum rate constant for solvent-mediated acid-base catalysis is about $10^{3} \mathrm{~s}^{-1}$; the maximum turnover numbers (catalytic rate constants) observed do not exceed this value for most enzymes ${ }^{40}$.

Acid-base catalysis need not be mediated by water. Kinetic studies of proton transfer between many different acids and bases have been made ${ }^{39}$. The over-all reaction can be written as

$$
\mathrm{DH}+\mathrm{A} \rightleftharpoons \mathrm{HA}+\mathrm{D}
$$

where $\mathrm{D}$ and $\mathrm{A}$ denote proton donor and acceptor, respectively. If the $\mathrm{p} K$ of the acceptor is much higher than that of the donor, the proton transfer to the acceptor is diffusion-controlled. The rate constant for the reverse reaction of equation (8) is then proportional to the equilibrium constant for the reaction, the ratio of the ionization constants of acceptor and donor, $K_{\mathrm{A}} / K_{\mathrm{D}}$. In terms of the intramolecular proton transfer which occurs after the donor and acceptor have diffused together, the specific rate constant for proton transfer in the forward direction must be about $10^{12} \mathrm{~s}^{-1}$ (much larger than the rate constant for diffusion apart of the reactants) and the rate constant for proton transfer for the reaction in the reverse direction must be approximately $10^{12}$ $\mathrm{K}_{\mathrm{A}} / K_{\mathrm{D}} \mathrm{s}^{-1}$.

Superficially, then, it would appear as though the upper limit for the maximum catalytic rate of an enzymatic reaction were $10^{12} \mathrm{~s}^{-1}$, but this is not the case. First, a catalytic cycle requires both protonation and deprotonation, and the rate cannot be maximal for both cases. Second, most substrates are very poor proton acceptors or donors, so that proton transfer from or to ionizable groups on the enzyme will be much slower than the maximum possible rate of proton transfer. Rates of proton transfer are considerably slower than normal for carbon acids and bases because of changes in electronic structure accompanying protonation and deprotonation ${ }^{39}$. The consequence of these limitations for enzymatic reactions is considerable. For example, if the $\mathrm{pK}$ difference between enzyme and substrate is seven $\mathrm{p} K$ units, the maximum proton transfer rate in the slowest direction would be about $10^{5} \mathrm{~s}^{-1}$. This is about the maximum turnover number observed for enzymes. The concentration of the intermediate formed would be only $10^{-7}$ of the enzyme concentration: this requires that the specific rate constant for further reactions of the intermediate must be greater than $10^{12} \mathrm{~s}^{-1}$, if an over-all turnover number of $10^{5} \mathrm{~s}^{-1}$ is to be achieved furthermore, because of the low concentration of the intermediate, it cannot be detected directly and its rate of appearance and disappearance cannot be studied directly. The maximum 
rate of an enzymatic reaction in this sample analysis is primarily determined by the $\mathrm{p} K$ difference between substrate and enzyme group. For almost all cases this difference is greater than seven $\mathrm{p} K$ units and the substrate is frequently a carbon acid or base, which further reduces the specific rate constants. In order to explain the observed rates of enzymatic reactions with this mechanism, the enzyme must considerably enhance the acidity or basicity of the substrate through interactions with specific protein groups.

In summary. on the basis of the above considerations. it is unlikely that the turnover number for enzymes will exceed about $10^{5} \mathrm{~s}^{-1}$, and it is also unlikely that it will be possible to detect the intermediates in acid-base catalysis because they are present in very small concentrations. Experimental results support these conclusions thus far.

A final mechanistic possibility which should be considered for acid-base catalysis is concerted proton transfers - that is, simultaneous proton acceptance and donation by the substrate. Unfortunately, the rate of such a process is difficult to estimate. The primary effect of a concerted process is to eliminate the necessity of forming an unstable reaction intermediate in very low concentrations. The upper bound for such a process can be taken as the direct rate of proton transfer between the acid and base groups on the enzyme involved in the catalysis. A typical $\mathrm{p} K$ difference is about two units, so the upper bound for the rate constant is about $10^{10} \mathrm{~s}^{-1}$. This is certainly unrealistically high, because of the generally poor acid-base properties of the substrate. In the most favourable model systems involving carbon acids and bases the proton transfer rates are reduced by three to four orders of magnitude. Thus, a specific rate constant of $10^{6} \mathrm{~s}^{-1}$ is a reasonable upper bound for the turnover number of enzymes involving concerted proton transfers.

For both concerted and sequential proton transfer mechanisms the observed turnover numbers for most enzymes are surprisingly close to the estimated upper bounds of the rate constants for proton transfer reactions. Thus, the elementary steps of proton transfer appear to be proceeding at close to their maximum possible rates for most enzymes.

A number of studies have been made of enzyme mechanisms with fast reaction techniques, and some general conclusions can be derived (cf. references 8 and 9). First, as discussed above, the initial formation of enzymesubstrate complexes is generally quite specific and rapid falmost diffusioncontrolled). Second, some type of cooperative isomerization or conformational change very often occurs following the bimolecular formation of enzyme-substrate complexes. This step probably involves reorienting the substrate (or enzyme) so as to produce effective catalysis. Third, a large number of reaction intermediates of comparable stability are frequently observed in enzymatic reactions. Many of the observed interconversions of reaction intermediates do not reflect the primary event of covalent bond formation or breakage. This implies that many of the chemical intermediates are present in concentrations too small to be detected by available techniques, which is consistent with the previous discussion of proton transfer reactions. In fact, the reason the over-all reaction is slow compared with proton transfer rates may be due to the reaction intermediates being present in very small concentrations.

The information discussed above can be used to form a plausible picture of 
how enzyme catalysis occurs. The enzyme appears to break down the catalysis into a number of steps, with the enzyme optimizing its configuration for each step. The actual chemical events occur at close to their maximal possible rates through the entire catalytic cycle, and the enzyme adapts its configuration through cooperative conformational changes, so that it can catalyse each of the elementary reactions very efficiently. The flexible structure of the enzyme, which is due to its macromolecular nature, permits it to be a good catalyst for all reaction steps and may explain why all enzymes are macromolecules.

\section{RIBONUCLEASE}

As an example of the elucidation of the elementary steps in enzyme catalysis the mechanism of action of bovine pancreatic ribonuclease A is now considered. This enzyme catalyses the breakdown of ribonucleic acid in two steps, as shown in Figure 1. First, the diester linkage is broken and a pyrimi-

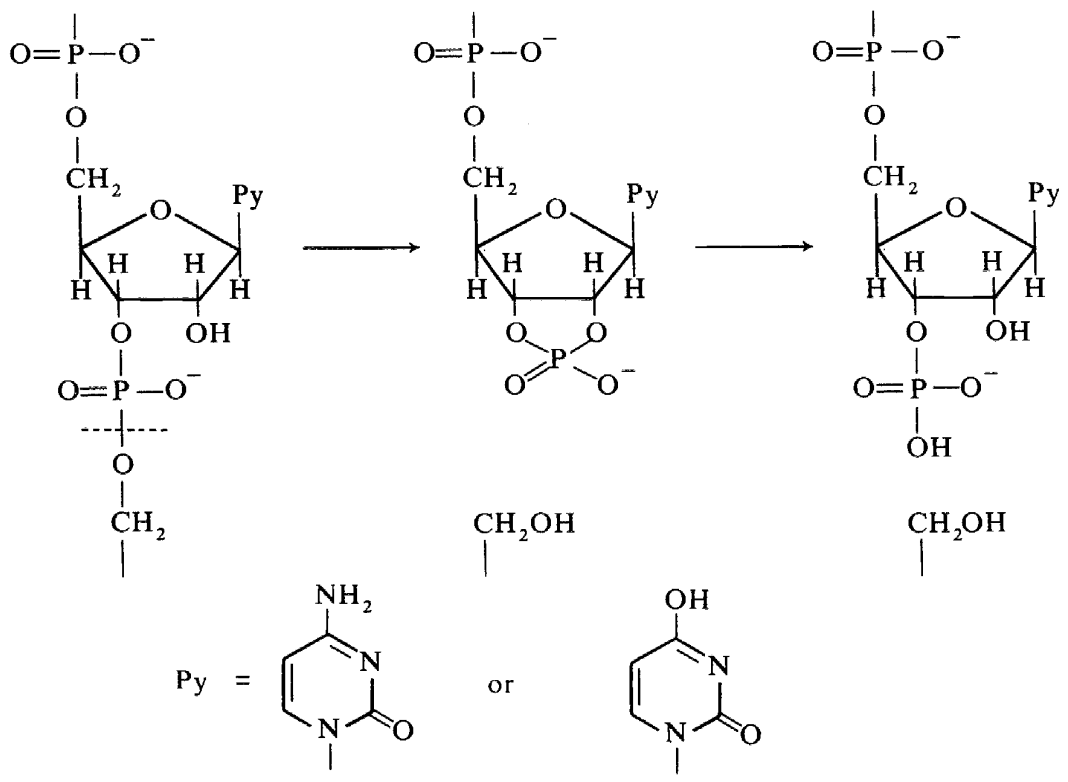

Figure 1. The two-step hydrolysis of ribonucleic acid catalysed by the enzyme bovine pancreatic ribonuclease $\mathrm{A}$

dine-2' $3^{\prime}$ cyclic phosphate is formed, and then the cyclic phosphate is hydrolysed to give the pyrimidine-3'-monophosphate and purine oligonucleotides with a terminal pyrimidine $3^{\prime}$-phosphate. Ribonuclease has been extensively studied by many methods: the amino acid sequence is known ${ }^{41-43}$, the three-dimensional structure is known ${ }^{44,45}$ and many other chemical and physical studies have been carried out with this enzyme (cf. reference 46).

Kinetic studies generally have not employed ribonucleic acid itself as a 
substrate, because the system becomes inhomogeneous as ribonucleic acid is degraded and the kinetic analysis is very complex. Instead model substrates of known structure, such as dinucleosides, pyrimidine- $2^{\prime}, 3^{\prime}$ cyclic phosphates and pyrimidine-3'-monophosphates, have been frequently used. The reaction can be conveniently divided into three states, corresponding to the three types of model compounds. This is possible because the reactions separating the three substances occur slowly relative to the rates characterizing the enzyme-substrate interactions. However, at equilibrium essentially only pyrimidine- $3^{\prime}$-monophosphates are present ${ }^{47,48}$.

In the absence of substrates, a relaxation process is observed in solutions of ribonuclease having a relaxation time in the range of $0.1-1 \mathrm{~ms}^{49}$. This is due to an isomerization of the enzyme. A simple mechanism consistent with the data is

$$
\mathrm{E}^{\prime} \mathrm{H} \rightleftharpoons \mathrm{EH} \rightleftharpoons \mathrm{E}+\mathrm{H}^{+}
$$

where $\mathrm{E}$ and $\mathrm{E}^{\prime}$ represent different enzyme conformations and the protolytic equilibrium is assumed to equilibrate rapidly relative to the interconversion of $\mathrm{E}^{\prime} \mathrm{H}$ and $\mathrm{EH}$. Although the exact nature of this isomerization is not known, the associated rate constants are considerably smaller in $\mathrm{D}_{2} \mathrm{O}$ than in $\mathrm{H}_{2} \mathrm{O}$ and the relaxation process is eliminated by modification of or binding at the active site of the enzyme. Therefore, a conformational change associated with the active site, possibly involving hydrogen bonding. seems likely.

A plausible explanation of this isomerization can be made in terms of the three-dimensional structure of the enzyme. Ribonuclease is a compact kidney-shaped molecule with the active site located along a groove ${ }^{44,45}$. Inhibitors are bound to the enzyme near two histidine residues (numbers 12 and 119 of the amino acid sequence). Chemical ${ }^{50,51}$ and n.m.r. ${ }^{52}$ evidence also suggest that these residues are at the catalytic site. At the top of the 'hinge' of the groove is a third imidazole residue (histidine 48). The imidazole ring is partially buried, and its environment could be altered by an opening and closing of the groove associated with the active site. A possible interpretation is that this observed relaxation process is associated with an opening and closing of the groove such that the imidazole residue is 'buried' in $E^{\prime} H$ and has a $\mathrm{p} K$ of 6.1 when exposed in the $\mathrm{EH}$ isomer.

The interaction of dinucleosides, pyrimidine-2', $3^{\prime}$-cyclic phosphates or pyrimidine- $3^{\prime}$-phosphates with the enzyme is characterized by two relaxation processes, in addition to the process associated with the unliganded enzyme $^{11-14}$. In all cases the results obtained can be described by a two-step mechanism : a bimolecular combination of enzyme and substrate followed by an isomerization or conformational change of the enzyme-substrate complex :

$$
\mathrm{E}+\mathrm{S} \rightleftharpoons \mathrm{X}_{1} \rightleftharpoons \mathrm{X}_{2}
$$

The rate constants associated with the first step for a variety of substances are included in Table 2; the rate constants for the second step are approximately $10^{3}-10^{4} \mathrm{~s}^{-1}$.

Many of the rate constants have been determined as a function of $\mathrm{pH}$ and temperature. The $\mathrm{pH}$ dependence of the bimolecular rate constant suggests that two ionizable groups on the enzyme are involved in the binding process, one in its basic form, the other in its acid form, with associated $\mathrm{p} K$ values of 
approximately 5.4 and $6.4^{11}$. This is consistent with $\mathrm{pK}$ values determined from steady state kinetic studies ${ }^{48,53,54}$. The $\mathrm{pH}$ dependence of the rate of the conformational change of both liganded and unliganded enzyme strongly suggests that a $\mathrm{p} K$ of approximately 6 is of importance in the relaxation process.

The simplest interpretation of these results is that the ionizable groups on the enzyme influencing the bimolecular rate constant are the imidazole rings of histidines 12 and 119 , since these have been directly implicated in the catalytic mechanism by chemical studies. The third ionizable group, with a $\mathrm{p} K$ of 6 , is again probably the imidazole residue of histidine 48 , and the conformational change is probably similar in nature to that associated with the unliganded enzyme. Direct evidence supporting this role of histidine 48 is found from n.m.r. studies which indicate that the binding of a $3^{\prime}$ nucleotide perturbs the environment of this imidazole residue ${ }^{52}$. The suggestion also has been made that the conformational change associated with substrate binding swings lysine 41 near the substrate to aid in the catalytic reaction.

Thus, the over-all mechanism for the enzymatic reaction might be constructed as follows. The enzyme exists in dynamic equilibrium between two forms, differing in the structure of the active site groove. The substrate is bound at a rate almost as fast as that at which it can diffuse to the active site. (This is derived from the magnitudes of the bimolecular rate constants in Table 2.) When the substrate binds, the groove shape is altered and lysine 41 swings over to the substrate to assist in the binding process, and the substrate is oriented very precisely so that the imidazole residues (histidines 12 and 119) can catalyse the chemical reaction through rapid proton transfer reactions. The conformational change is then reversed and the product dissociates. Both the transesterification and hydrolysis steps proceed in a similar manner. This mechanism is shown pictorially in terms of the enzyme structure in Figure 2.

Unfortunately, the elementary steps associated with the proton transfer reactions cannot be studied: only the over-all rate of the conversion of substrate to product (and vice versa) in the active site can be determined. This varies from about 10 to $10^{4} \mathrm{~s}^{-1}$ for a variety of substrates at their optimal $\mathrm{pH}^{48,53,54}$. The reason that the elementary steps cannot be observed is undoubtedly that the concentrations of the reaction intermediates are too small. Although the details of the proton transfer process remain to be elucidated, detailed consideration of the three-dimensional structure and stereochemical studies indicate that the mechanism probably involves a concerted proton transfer between two imidazole residues and the substrate such as that shown in Figure $2^{5-58}$.

Thus, a combination of detailed kinetic, chemical, and structural studies has led to a fairly complete picture of the catalytic process for ribonuclease and has come close to resolving the entire time course of the reaction into its elementary steps.

\section{REGULATION}

Although the regulation of enzyme activity by switching the enzyme between active and inactive forms has features in common with catalysis, namely 


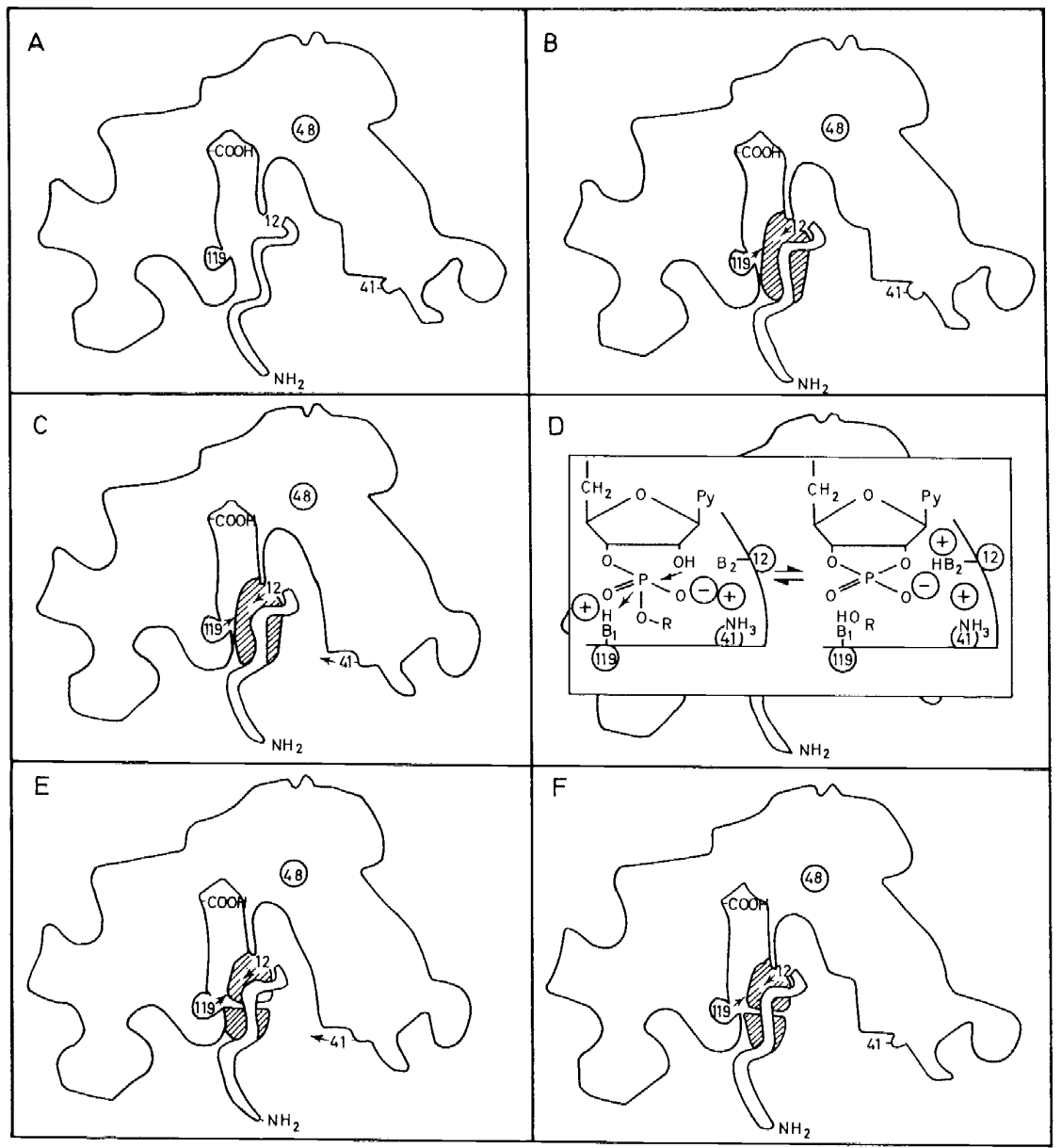

Figure 2. A pictorial representation of the first half of the ribonuclease reaction. The free enzyme (A) can exist in two conformations differing by small movements about the hinge region joining the two halves of the molecule; the substrate is bound (B) and a conformational change occurs bringing Lys 41 close to the active site $(\mathrm{C})$; acid-base catalysis occurs (D); products are formed (E); a conformational change occurs $(F)$; and the product dissociates to give the free enzyme (A)

ligand binding and conformational changes, a number of distinct differences in the elementary steps exist. The most important new element is that regulatory enzymes almost always contain more than a single polypeptide chain or subunit. The interactions between subunits, which may be essentially identical or quite different in structure, is the controlling feature of most regulatory mechanisms, but is generally of little consequence in catalysis.

Regulatory processes can be most easily studied by observing the dependence of the reaction rate on the substrate and effector concentrations (and in some cases the enzyme concentration). For simple enzymes a plot of the steady state initial velocity versus substrate concentration is hyperbolic, as shown in Figure 3. This already provides primitive regulation, since the 


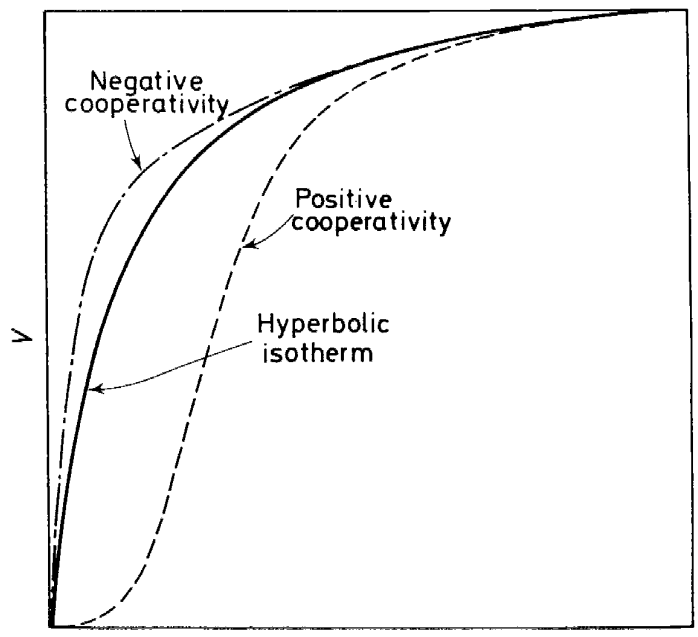

[S]

Figure 3. A schematic plot of the steady state initial velocity, $V$, versus the substrate concentration, [S]. The three curves represent isotherms for a hyperbolic saturation function (-.-), positive cooperativity (- - - ) and negative cooperativity $(-\cdot-)$

reaction rate is regulated by the level of the substrate concentration, and the initial velocity reaches a limiting value at high substrate concentrations. This limiting rate, the maximal velocity, is usually proportional to the total concentration of enzyme. For regulatory enzymes the initial velocity-substrate isotherms are frequently non-hyperbolic. In some cases the isotherms are sigmoidal and in other cases the velocity increases very rapidly initially as the substrate concentration increases and then much more slowly at high substrate concentrations (see Figure 3). The former case is termed positive cooperativity, since the substrate apparently binds to the enzyme with increasing strength as the substrate concentration increases; therefore, very small changes in substrate concentration produce large changes in rate, providing a regulation of enzyme activity. The latter case is termed negative cooperativity, since the binding between substrate and enzyme apparently weakens as the substrate concentration increases; this has the effect of broadening the range of concentration over which regulation can occur. In both cases multiple binding sites on the enzyme must exist, and the interactions occurring between binding sites for identical ligands are called homotropic interactions ${ }^{59}$.

Metabolic effectors, activators and inhibitors are structurally dissimilar to substrates, which, coupled with other evidence, has led to the now generally accepted proposal that effectors exert regulatory control over catalysis by reacting at an allosteric site quite distinct from the catalytic site (heterotropic interactions) ${ }^{59}$. Allosteric interactions are defined as indirect interactions between topographically distinct sites mediated by the protein molecules through conformational changes and/or subunit interactions. These interactions can induce or stabilize conformational states which either have a different affinity for the substrate ( $\mathrm{K}$ system) or have a different catalytic potential (V system) or both ${ }^{59}$. For $\mathrm{K}$ systems an inhibitor generally causes 
the initial velocity-substrate isotherm to become more sigmoidal, while an activator causes it to be less sigmoidal, as illustrated in Figure 4. In both cases the same maximal velocity is attained at sufficiently high substrate concentrations, and the inhibitors and activators provide regulation only over a restricted range of substrate concentrations. For $\mathrm{V}$ systems the maximal velocity is increased by an activator and decreased by an inhibitor.

A number of molecular models have been proposed to account for the regulation of enzyme activity. All of these models are based on the subunit structure of proteins and alterations in subunit interactions and/or conformations coupled to ligand binding. Two limiting molecular models are often used to describe the alteration of enzyme activity through conformational changes linked to ligand binding. One is due to Monod, Wyman and Changeux (MWC model) ${ }^{59}$; the other is due to Adair, Koshland, Nemethy and Filmer (AKNF model $^{60,61}$.

The MWC model is based on three postulates: (1) the enzyme consists of two or more identical subunits, each containing a site for the substrate or effector; (2) at least two different conformational states (usually designated as $\mathrm{R}$ and $\mathrm{T}$ states) are in equilibrium and differ in their affinities for substrate and/or effector; and (3) the conformational changes of all subunits occur in a concerted manner. A schematic illustration of the MWC model for a foursubunit enzyme is shown in Figure 5, where squares and circles are used to indicate different enzyme conformations. In the absence of substrate, the enzyme exists largely in $\mathrm{T}$ states (the square conformation), but substrates bind preferentially to the $\mathrm{R}$ states (the circular conformation), so that the conformational equilibrium is shifted to the $R$ states by the binding of substrate. Quantitative analysis of this model indicates that sigmoidal binding isotherms can be generated. Activators and inhibitors, by binding preferentially to the $\mathrm{R}$ and $\mathrm{T}$ states, respectively, can reduce or enhance the sigmoidicity of the binding isotherms, exactly as often found for $\mathrm{K}$ systems (Figure 4).

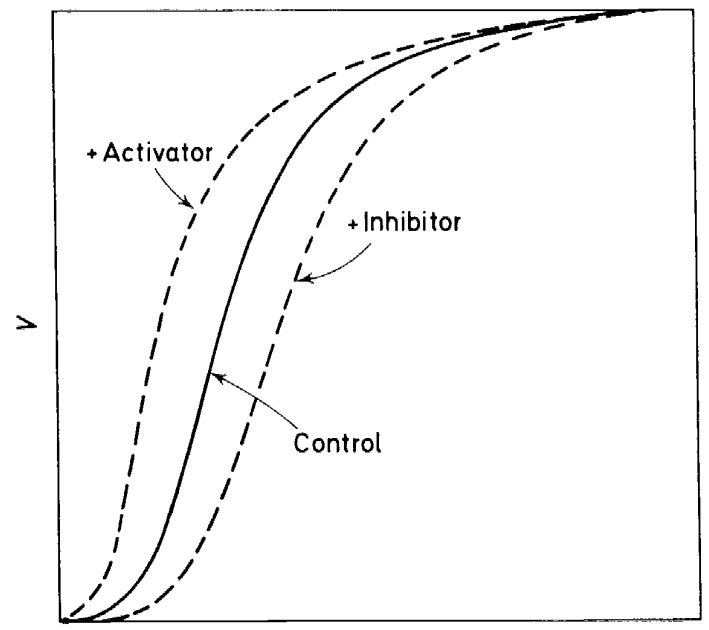

[S]

Figure 4. The effect of an activator and inhibitor on the initial velocity-substrate isotherm for a $\mathrm{K}$ system with positive cooperativity 
In the MWC model the subunits are all in the same conformation-that is, hybrid çonformational states containing both squares and circles canmot exist. An important limitation of this model is that only positive cooperativity can occur, so that a basis for negative cooperativity is not provided.

The basic assumptions of the AKNF model are that (1) two conformational states are available to each subunit, (2) only the subunit to which the ligand is bound changes its conformation and (3) the ligand-induced conformational change in one subunit alters its interactions with neighbouring subunits. The strength of the subunit interactions may be increased or decreased or remain the same. A schematic illustration of this model for a four-subunit protein is included in Figure 5. Because each liganded state has different subunit interactions, it has a different effective binding constant for adding another ligand. Thus, this model can readily generate substrate binding isotherms displaying positive or negative cooperativity, or even both. Activators and inhibitors

Concerted model (MWC)

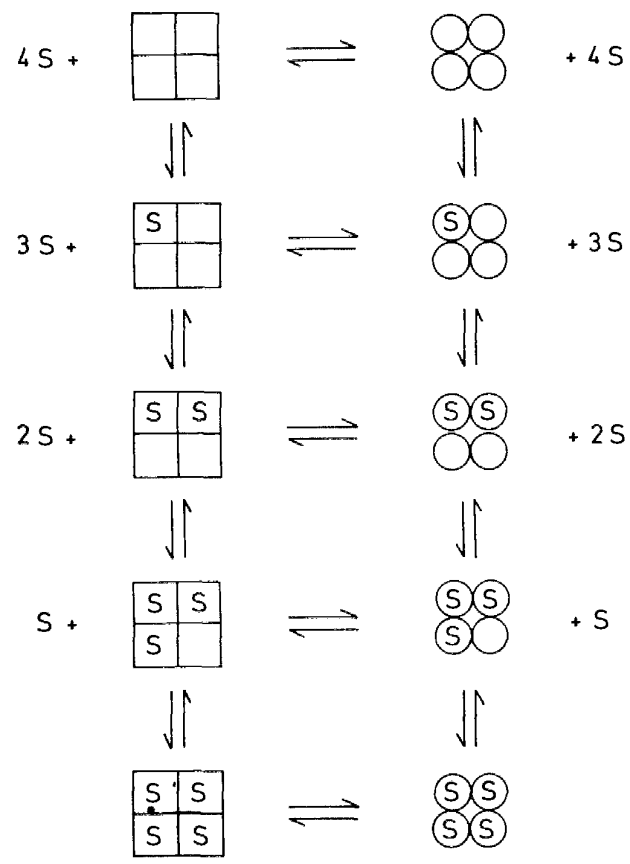

Simple sequential model (AKNF)

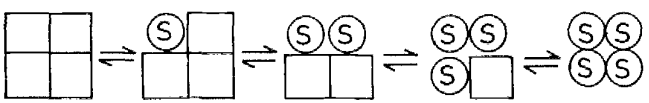

Figure 5. Schematic representations of the MWC and AKNF models for a four-subunit enzyme. The squares and circles designate different subunit conformations and $\mathrm{S}$ is the substrate. The free substrate has been omitted from the AKNF model for the sake of simplicity 
can alter the effective substrate binding constants by changing the subunit interactions. In contrast to the concerted nature of the MWC model, the AKNF model predicts a strictly sequential change of subunit conformations exactly paralleling ligand binding. Clearly a more general model can be generated by permitting both sequential and partially concerted conformational changes. In practice these models are often very difficult to distinguish by experimental measurements.

The models discussed thus far are equilibrium models in that alterations in the rates of enzyme catalysis are explained by changes in the equilibrium binding characteristics of the enzyme. For $\mathrm{K}$ systems such an analysis is appropriate if the binding steps and conformational changes are rapid relative to the rate-determining step in catalysis. These models can also be used for $\mathrm{V}$ systems, with the additional postulate that each of the conformational states of the enzyme has a different turnover number. The equilibrium assumption appears to be valid for many systems. However, it should be noted that apparent cooperativity in initial velocity-substrate isotherms can be generated by parallel kinetic pathways and special relationships between the rate constants $^{62-64}$. In other words, complex mechanisms can lead to apparent cooperativity without postulating special conformational transitions. Although this possibility exists, thus far it has not been shown that this mechanism is utilized by regulatory enzymes.

An extreme alteration of subunit interactions occurs in polymerizationdepolymerization reactions, and polymerization equilibria probably play an important role in the regulation of some enzymes ${ }^{65}$. If, for example, an enzyme exists in two polymeric states, each having a different affinity for substrate and effectors, a model is generated analogous to the MWC model, except that cooperativity in the binding isotherm is also dependent on the enzyme concentration ${ }^{66,67}$. Again only positive cooperativity can be generated with this model. Both $\mathrm{K}$ systems and $\mathrm{V}$ systems can be obtained with this model, exactly as previously discussed for conformational models, provided the polymerization equilibria are adjusted rapidly relative to the rate-determining step of catalysis.

Thus far the assumption has been made that allosteric enzymes respond rapidly to changes in ligand concentration. However, this is not required on a functional basis. In fact, systems are known where ligands can induce changes in enzyme activity much more slowly than the rate of the over-all catalytic reaction. This causes a time lag in the response of the enzyme to changes in the ligand concentrations. Such slowly responding enzymes are called 'hysteretic' ${ }^{68}$. The molecular basis for this mode of regulation apparently is not fundamentally different from previously discussed models : slow conformational changes, slow polymerization-depolymerization of enzymes and slow displacement of a tightly bound ligand have been proposed in specific cases.

Only the triggering of regulatory processes by ligand binding has been discussed. However, regulation can also occur through enzyme-catalysed covalent modification of an enzyme - for example by phosphorylation and adenylation $^{69}$.

The elementary steps in the regulatory models discussed are not fundamentally different from those generally involved in macromolecular confor- 
mational transitions and the binding of small molecules by proteins. The great range in the rates of regulatory processes must arise from differences in the degree of cooperativity in the conformational transitions (cf. Table 3). The fact that inter subunit conformational changes as well as intra subunit conformational changes occur also is relevant. A new step which may be of importance is the polymerization-depolymerization of macromolecules. The basic interactions involved are hydrogen bonding, solvation, hydrophobic, and electrostatic interactions, but essentially no quantitative rate data are available for appropriate polymerization equilibria. The dissection of enzyme regulatory processes into elementary steps is not quite as advanced as for enzyme catalysis, but nevertheless useful molecular models are available (cf. references 65 and $69-71$ for more extensive reviews).

\section{ASPARTATE TRANSCARBAMYLASE}

As mentioned earlier, aspartate transcarbamylase is a regulatory enzyme which catalyses the reaction in equation (11). This is the first committed step in the biosynthetic pathway for the synthesis of pyrimidines. The enzyme from<smiles>C[CH-]C(=O)C(CC(=O)[O-])NC(=O)NC(CC(=O)[O-])C(=O)[O-]</smiles>

Escherichia coli has been extensively studied by many workers, and a number of reviews are available ${ }^{65,72-74}$. The binding of aspartic acid to the enzyme in the presence of a saturating concentration of carbamyl phosphate, as measured by initial velocities, has a sigmoidal binding isotherm. The sigmoidicity of this isotherm is increased by the feedback inhibitor CTP and decreased by the activator ATP, exactly as shown in Figure $4^{4}$. The maximum velocity is not altered by effectors.

The enzyme can be resolved into two types of subunits: a catalytically active subunit not subject to nucleotide control and a catalytically inactive subunit that binds CTP strongly ${ }^{75}$. These two types of subunits can be readily reconstituted to give an active enzyme subject to control by nucleotides. Thus, the allosteric nature of the control process is clearly established. The native enzyme contains six identical catalytic polypeptide chains and six identical regulatory polypeptide chains with six regulatory and six catalytic sites ${ }^{76-81}$. The catalytic subunit is a trimer and the regulatory subunit is primarily a dimer. Electron microscopy and x-ray studies have established the general nature of the three-dimensional structure of the enzyme: the two catalytic trimers are connected by the regulatory dimers, with no direct interaction occurring between the catalytic trimers ${ }^{82,83}$. The native molecule has a threefold and a twofold symmetry axis. A very schematic model of this structure is shown in Figure 6.

The binding of nucleotide effectors to the enzyme is complex. The binding isotherms indicate negative cooperativity in binding to regulatory sites, as well as binding to the catalytic sites which can be eliminated by millimolar 


\section{ELEMENTARY STEPS IN ENZYME CATALYSIS AND REGULATION}
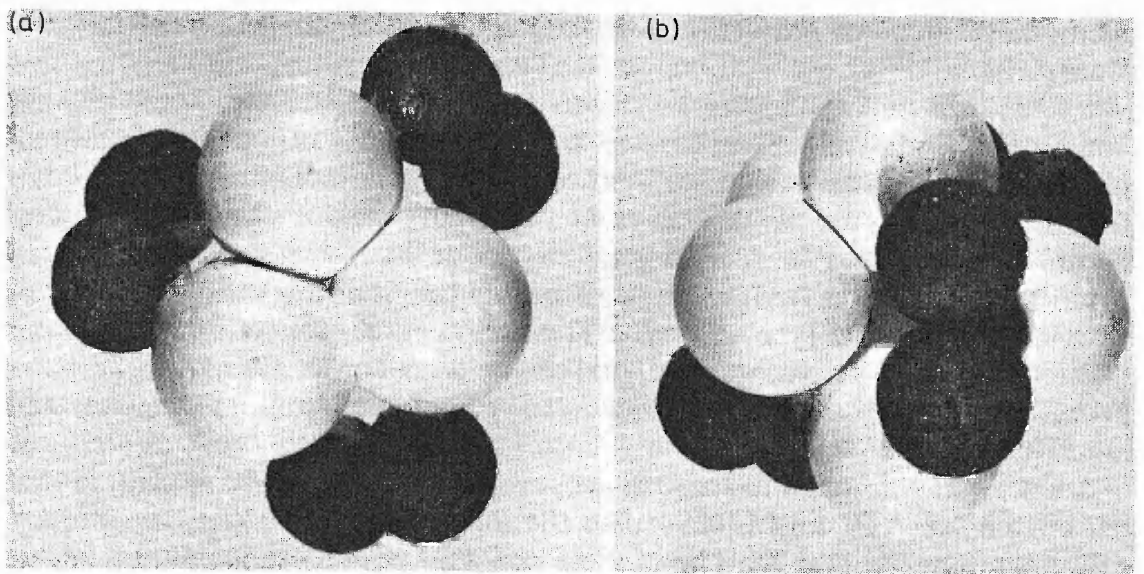

Figure 6. A pictorial representation of the structure of aspartate transcarbamylase. The lightcoloured portions are catalytic subunits and the dark-coloured portions are regulatory subunits.

carbamyl phosphate $79.81,84-86$. The cooperative unit is the regulatory dimer: binding of an effector molecule to one dimer site considerably reduces the binding affinity for the second effector molecule. The inhibition and activation are directly proportional to the fraction of total regulatory sites occupied, so that all regulatory sites participate equally in the regulatory function ${ }^{81,86}$. The initial velocity-aspartate isotherm is sigmoidal, and the equilibrium binding of succinate, an aspartate analogue, in the presence of carbamyl phosphate also displays a sigmoidal binding isotherm ${ }^{87}$. Therefore, this enzyme utilizes both positive and negative cooperativity in its regulatory mechanism.

Extensive kinetic studies have been made of the binding of ligands to aspartate transcarbamylase by temperature jump and stopped flow techniques. Effector molecules studied include CTP ${ }^{20}$, 5-bromo-CTP ${ }^{19.86}$. 5-bromo-CDP ${ }^{86}$, 5-bromo-CMP ${ }^{86}$ and the ATP analogue, 6-mercapto-9- $\beta$ D-ribofuranosyl-purine- $5^{\prime}$-triphosphate ${ }^{88}$; the kinetics of the binding of carbamyl phosphate and the aspartate analogue, succinate, also have been studied. Elementary steps associated with both catalysis and regulation have been observed. A summary of the results obtained is presented in Table 6; only the

Table 6. Elementary steps in catalysis and regulation for aspartate transcarbamylase

Reactant

Carbamyl phosphate

(+ succinate)

Succinate or L-malate (+ carbamyl phosphate)

CTP, ATP analogues
Mechanism

Bimolecular association

Conformational change (stepwise)

Conformational change (concerted)

Bimolecular association

Conformational change (concerted)

Bimolecular association (stepwise)

Function

Binding

Catalysis

Regulation

Binding

Regulation

Binding

Regulation 
elementary steps of importance in the regulatory mechanism will be considered here.

The equilibrium and kinetic data for the binding of all effector molecules are consistent with a simple mechanism in which a rate-limiting conformational change follows a relatively rapid bimolecular reaction. The same conformational change occurs at all classes of regulatory sites and alters the interactions between subunits. The negative cooperativity occurs in the initial rapid binding step. In terms of the molecular models discussed earlier, this is a simplified sequential type of model. The same two conformational states are utilized by both activators and inhibitors, since only a single relaxation process is observed with the enzyme in the presence of both an activator and inhibitor.

A simple regulatory mechanism accommodating all available data is that the binding of ATP and CTP causes the formation of two enzyme-effector conformations, $X_{1}$ and $X_{2}$. The binding of CTP favours the formation of one conformation (say $\mathrm{X}_{1}$ ), which can be regarded as the 'off' state, while the binding of ATP favours the formation of the other conformation $\left(\mathrm{X}_{2}\right)$, which can be regarded as the 'on' state. As expected the binding of carbamyl phosphate and succinate is also found to favour the formation of $X_{2}$.

The binding of succinate to the enzyme in the presence of saturating concentrations of carbamyl phosphate is quite complex. Two relaxation processes are observed with the isolated catalytic subunit which can be explained in terms of a bimolecular binding step followed by a conformational change of importance in catalysis ${ }^{18}$. These same relaxation processes are observed with the native enzyme and are not altered by the binding of effectors. In addition a new relaxation process is observed with the native enzyme ${ }^{21}$. The concentration dependence of the associated relaxation time (which is in the range of about $2-20 \mathrm{~ms}$ ) can be analysed quantitatively in terms of a concerted conformational change, analogous to the MWC model. This conformational transition is distinct from the conformational change associated with effector binding, since both transitions are found to occur simultaneously. Moreover, activators and inhibitors alter the concentration dependence of the relaxation time exactly as predicted by the concerted model. Also, the kinetic parameters derived can be used to generate a sigmoidal equilibrium binding isotherm.

The interaction of carbamyl phosphate with the native enzyme at high concentrations of succinate also is somewhat complex; a relatively slow relaxation process, with a relaxation time of $10-100 \mathrm{~ms}$, is found to accompany the binding of carbamyl phosphate to the native enzyme in the presence of succinate, but is not observed with the catalytic subunit ${ }^{22}$. The simplest mechanism consistent with the data is a concerted conformational transition, which appears to be distinct from the one associated with succinate binding, since the concentration dependence is different, and the binding of CTP alters the relaxation time differently in the two cases.

In addition to the kinetic data cited above, a large number of chemical and physical studies have provided evidence that conformational changes accompany ligand binding (cf. reference 73). The methods utilized include ultra-violet difference spectroscopy, optical rotatory dispersion, ultracentrifugation, trypsin digestion of the enzyme, measurement of the rate 
of reaction of enzyme sulphhydryl groups and sodium dodecyl sulphate inactivation of the enzyme. The results obtained indicate that the binding of effector molecules causes a different conformational change from that caused by the binding of substrates and that the binding of succinate to the native enzyme causes a much larger conformational change than binding to the isolated catalytic subunit.

The over-all control mechanism for aspartate transcarbamylase can be depicted as follows. The effector molecules, ATP and CTP, carry out their function by altering a two-state conformational equilibrium, which occurs roughly independently in each regulatory chain. The local conformational changes occurring in the regulatory chain alter its interaction with the catalytic subunit, thereby altering the enzyme activity. Local conformational changes involved in the catalytic process also occur in the catalytic subunit when carbamyl phosphate and succinate bind. The conformational changes involved in control which are induced by carbamyl phosphate and succinate binding appear to be quite distinct from each other and from that induced by CTP and ATP binding. These two conformational transitions appear to be concerted in nature. Athough the structural basis of the concerted conformational transitions is not yet known, rotation of the catalytic subunits with respect to each other around the threefold symmetry axis may be involved (cf. Figure 6) ${ }^{72}$. In any event, the over-all control mechanism appears to be a combination of several different conformational transitions, each of which can lead to inhibition or enhancement of enzymic activity. This multiplicity and coupling of conformational changes, which provides a sensitive and versatile control mechanism, is somewhat analogous to a mini-computer control of the enzymatic reaction with macromolecular conformational changes being utilized as interlocking switches.

\section{CONCLUSION}

The general approach to enzyme catalysis and regulation emphasized, namely the time resolution of the reaction mechanisms into their elementary steps, provides insight into the molecular basis of the mechanisms. Of necessity, only a limited number of systems were discussed. This brief discussion is intended to illustrate the potential of this approach and the information which can be obtained.

\section{ACKNOWLEDGEMENT}

This work was supported by a grant from the National Institutes of Health (GM 13292).

\section{REFERENCES}

C. Frieden and R. A. Alberty, J, Biol. Chem. 212, 859 (1955).

2 L. T. Rozelle and R. A. Alberty, J. Phys. Chem. 61, 1637 (1957).

${ }^{3}$ L. E. Erickson and R. A. Alberty, J. Phys. Chem. 63, 705 (1959).

${ }^{4}$ J. C. Gerhart and A. B. Pardee, J. Biol. Chem. 237, 891 (1964).

5 J. V. Pasonneau and O. H. Lowry, Biochem. Biophys. Res. Commun. 10, 7 (1962).

' J. V. Pasonneau and O. H. Lowry. Biochem. Biophys. Res. Commun. 13. 372 (1963). 
7 Investigation of Rates and Mechanisms of Reactions. Vol. 6, Part II: 'Investigation of elementary steps in solution and very fast reactions', G. G. Hammes, ed. in Techniques of Chemistry, A. Weissberger, series ed. Wiley-Interscience; New York (1974).

${ }^{8}$ G. G. Hammes, Adv. Prot. Chem. 23, 1 (1968).

9 G. G. Hammes and P. R. Schimmel, The Enzymes, 2, 67 (1970).

10 G. P. Hess, J. McConn, E. Ku and G. McConkey, Phil. Trans. Roy. Soc. B, 257, 89 (1970).

11 G. G. Hammes and F. G. Walz, Jr, J. Amer. Chem. Soc. 91, 7197 (1969).

12 J. E. Erman and G. G. Hammes, J. Amer. Chem. Soc. 88, 5067 (1966).

13 E. J. del Rosario and G. G. Hammes, J. Amer. Chem. Soc. 92,1750 (1970).

14 J. E. Erman and G. G. Hammes, J. Amer. Chem. Soc. 88, 5614 (1966).

15 G. G. Hammes and J. K. Hurst, Biochemistry, 8, 1083 (1969).

16 H. de A. Heck, J. Biol. Chem. 244, 4375 (1969).

17 K. Kirschner, E. Gallego, I. Schuster and D. Goodall, J. Mol. Biol. 58. 29 (1971).

18 G. G. Hammes, R. W. Porter and G. R. Stark, Biochemistry, 10, 1046 (1971).

19 J. Eckfeldt, G. G. Hammes, S. C. Mohr and C.-W. Wu, Biochemistry, 9. 3353 (1970).

20 L. W. Harrison and G. G. Hammes, Biochemistry, 12, 1395 (1973).

21 G. G. Hammes and C.-W. Wu, Biochemistry, 10, 1051 (1971).

22 G. G. Hammes and C.-W. Wu, Biochemistry, 10, 2151 (1971).

23 E. D. Barber and H. J. Bright, Proc. Nat. Acad. Sci. US, 60, 1370 (1968).

${ }_{24}$ G. W. Hatfield and H. E. Umbarger, J. Biol. Chem. 245, 1742 (1970).

25 W. Maier, Z. Elektrochem. 64, 132 (1960).

${ }^{26}$ L. Borucki, Ber. Bunsenges. Physik. Chem. 71, 504 (1967).

27 G. G. Hammes and A. C. Park, J, Amer. Chem. Soc. 91, 956 (1969).

28 G. G. Hammes and H. O. Spivey, J. Amer. Chem. Soc. 88, 1621 (1966).

29 G. G. Hammes and P. J. Lillford, J. Amer. Chem. Soc. 92,7578 (1970).

${ }^{30}$ E. Grunwald and E. K. Ralph, III, J. Amer. Chem. Soc. 89, 4405 (1967).

31 G. G. Hammes and W. Knoche, J. Chem. Phys. 45, 4041 (1966).

32 G. G. Hammes and N. C. Pace, J. Phys. Chem. 72, 2227 (1968).

${ }^{33}$ G. G. Hammes and T. B. Lewis, J. Phys. Chem. 70, 1610 (1966).

${ }^{34}$ G. G. Hammes and P. B. Roberts, J. Amer. Chem. Soc. 90, 7119 (1968).

35 G. G. Hammes and J. C. Swann, Biochemistry, 6, 1591 (1967).

36 G. G. Hammes and P. R. Schimmel, J. Amer. Chem. Soc. 89, 442 (1967).

37 J. Applequist and P. Doty, Abstracts, 135th Meeting of American Chemical Society, Boston, April 5, 1959.

38 A. F. Barksdale and J. E. Stuehr, J. Amer. Chem. Soc. 94, 3334 (1972).

39 M. Eigen, Angew. Chem. 75, 489 (1963).

40 M. Eigen and G. G. Hammes, Adv. Enzymol. 25, 1 (1963).

41 C. H. W. Hirs, S. Moore and W. H. Stein, J. Biol. Chem. 235, 633 (1960).

42 J. J. Potts, A. Berger, J. Cooke and C. B. Anfinsen, J. Biol. Chem. 237, 1851 (1962).

43 D. J. Smith, W. H. Stein and S. Moore, J. Biol. Chem. 238, 227 (1963).

44 G. Kartha, J. Bello and D. Harker, Nature, 213, 862 (1967).

45 H. W. Wyckoff, K. D. Hardman, N. M. Allewell, T. Ingami, L. N. Johnson and F. M. Richards, J. Biol. Chem. 242, 3984 (1967).

46 F. M. Richards and H. W. Wyckoff, The Enzymes, 4, 647 (1971).

47 J. T. Bahr, R. E. Cathou and G. G. Hammes, J. Biol. Chem. 240, 3372 (1965).

48 E. J. del Rosario and G. G. Hammes, Biochemistry, 8, 1884 (1969).

49 T. C. French and G. G. Hammes, J. Amer. Chem. Soc. 87, 4669 (1965).

50 A. M. Crestfield, W. H. Stein and S. Moore, J. Biol. Chem. 238, 2421 (1963).

51 R. Heinrikson, W. H. Stein and S. Moore, J. Biol. Chem. 240, 2921 (1965).

52 D. H. Meadows and O. Jardetzky, Proc. Nat. Acad. Sci. US, 61, 406 (1968).

53 D. G. Herries, A. P. Mathias and B. R. Rabin, Biochem. J. 85, 127 (1962).

54 H. Witzel, Prog. Nuc. Acid. Res, 2, 221 (1963).

55 D. A. Usher, D. I. Richardson Jr and F. Eckstein, Nature, 228, 663 (1970).

56 G. C. K. Roberts, E. A. Dennis, D. H. Meadows, J. S. Cohen and O. Jardetzky, Proc. Nat. Acad. Sci. US, 62, 1151 (1969).

57 D. Findlay, D. G. Herries, A. P. Mathias, B. R. Rabin and C. A. Ross, Biochem. J. 85, 152 (1962).

58 D. A. Usher, E. S. Erenrich and F. Eckstein, Proc. Nat, Acad. Sci. US. 69, 115 (1972).

59 J. Monod, J. Wyman and J.-P. Changeux, J. Mol. Biol. 12, 88 (1965). 


\section{ELEMENTARY STEPS IN ENZYME CATALYSIS AND REGULATION}

60 G. S. Adair, J. Biol. Chem. 63, 529 (1925).

61 D. E. Koshland Jr, G. Nemethy and D. Filmer, Biochemistry, 5, 365 (1966).

62 A. Worcel, S. Goldman and W. W. Cleland, J. Biol. Chem. 240, 3399 (1966).

63 B. D. Sanwal and R. A. Cook, Biochemistry, 5, 886 (1966).

64 J. R. Sweeny and J. R. Fisher, Biochemistry, 7, 561 (1968).

65 G. G. Hammes and C.-W. Wu, Adv. Biophys. Bioeng., 3, 1 (1974).

66 C. Frieden and R. Colman, J. Biol. Chem. 242, 1705 (1967).

67 L. W. Nichol, W. J. H. Jackson and D. J. Winzor, Biochemistry, 6, 2449 (1967).

${ }^{68}$ C. Frieden, J. Biol. Chem. 245, 5788 (1970).

69 E. R. Stadtman, The Enzymes, 1, 397 (1970).

70 D. E. Koshland Jr, The Enzymes, 1, 341 (1970).

71 K. Kirschner, Current Topics in Cellular Regulation, 3, 167 (1971).

72 J. C. Gerhart, Current Topics in Cellular Regulation, 2, 275 (1970).

73 G. R. Jacobson and G. R. Stark, The Enzymes, 9, 225 (1973).

74 G. G. Hammes and C.-W. Wu, Science, 172, 1205 (1971).

75 J. C. Gerhart and H. K. Schachman, Biochemistry, 4, 1054 (1965).

${ }^{76}$ K. Weber, Nature, 218, 1116 (1968).

77 G. G. Hammes, R. W. Porter and C.-W. Wu, Biochemistry, 9, 2292 (1970).

78 E. A. Meighen, V. Pigiet and H. K. Schachman, Proc. Nat. Acad. Sci. US, 65, 234 (1970).

79 C. C. Winlund and M. J. Chamberlin, Biochim. Biophys. Res. Commun. 40, 43 (1970).

80 J. P. Rosenbusch and K. Weber, J. Biol. Chem. 246, 1644 (1971).

81 S. Matsumoto and G. G. Hammes, Biochemistry, 12, 1388 (1973).

82 K. E. Richards and R. C. Williams, Biochemistry, 11, 3393 (1972).

83 S. H. Warren, B. P. Edwards, D. R. Evans, D. C. Wiley and W. N. Lipscomb, Proc. Nat. Acad. Sci. US, 70, 1119 (1973).

84 T. Buckman, Biochemistry, 9, 3255 (1970).

85 C. W. Gray, M. J. Chamberlin and D. M. Gray, J. Biol. Chem. 248, 6071 (1973).

86 C. Tondre and G. G. Hammes, Biochemistry, 13, 3131 (1974).

87 J.-P. Changeux, J. C. Gerhart and H. K. Schachman, Biochemistry, 7, 538 (1968).

88 C.-W. Wu and G. G. Hammes, Biochemistry, 12, 1400 (1973). 